\title{
Gastos catastróficos em saúde: análise da associação com condições socioeconômicas em Minas Gerais, Brasil
}

\author{
Catastrophic health expenditures: analysis of the association \\ with socioeconomic conditions in Minas Gerais, Brazil
}

Jéssica de Brito Macedo (https://orcid.org/0000-0002-7415-4462) ${ }^{1}$

Alexandra Crispim Boing (http://orcid.org/0000-0001-7792-4824) ${ }^{2}$

Juliana Mara Andrade (https://orcid.org/0000-0002-7480-519X) ${ }^{1}$

Helton Saulo (https://orcid.org/0000-0002-4467-8652) ${ }^{3}$

Rodrigo Nobre Fernandez (https://orcid.org/0000-0001-8596-2898) ${ }^{4}$

Fabíola Bof de Andrade (https://orcid.org/0000-0002-3467-3989) ${ }^{1}$

\footnotetext{
${ }^{1}$ Instituto René Rachou, Fundação Oswaldo Cruz (Fiocruz-Minas). Av. Augusto de Lima 1715, Barro Preto. 30190-002 Belo Horizonte MG Brasil. fabiola.bof@fiocruz.br ${ }^{2}$ Programa de PósGraduação em Saúde Coletiva, Universidade Federal de Santa Catarina. Florianópolis SC Brasil.

${ }^{3}$ Departamento de Estatística, Universidade de Brasília. Brasília DF Brasil. ${ }^{4}$ Departamento de Economia, Universidade Federal de Pelotas. Pelotas RS Brasil.
}

\begin{abstract}
This study aimed to assess catastrophic health expenditures (CHE) and its association with socioeconomic conditions in 2009, 2011 and 2013 in Minas Gerais, Brazil. A cross-sectional study was carried out with data from the Household Sample Survey. The dependent variable was the CHE in each year of the survey. Expenditures that exceeded $10 \%$ and 25\% of household income were considered catastrophic. The association between catastrophic health expenditure and independent variables was tested by the Poisson regression. The prevalence of $\mathrm{CHE}$ ranged from $9.0 \%$ to $11.3 \%$ and $18.9 \%$ to $24.4 \%$ within the limits of $10 \%$ and $25 \%$, and 2011 recorded the lowest values. The largest proportion of health expenditure (94\%) was related to the acquisition of medicines. The prevalence of CHE was lower among those responsible for the household with 12 or more years of study than those with no formal education. Households with a higher wealth score had, in both limits, lower prevalence of CHE than those of the first quintile. We concluded that health expenditures significantly affected the budget of households in Minas Gerais and the purchase of medicines was the main component of spending. The findings reinforce the role of the Brazilian Unified Health System (SUS) in minimizing CHE and reducing socioeconomic inequalities.
\end{abstract}

Key words Health expenditures, Socioeconomic factors, Health inequities
Resumo $O$ objetivo deste estudo foi avaliar os gastos catastróficos em saúde (GCS) e sua associação com condições socioeconômicas nos anos de 2009, 2011 e 2013 em Minas Gerais. Realizou-se um estudo transversal com dados da Pesquisa por Amostra de Domicílios. A variável dependente foi o GCS, em cada ano da pesquisa. Foram considerados catastróficos os gastos que ultrapassaram os limites de $10 \%$ e $25 \%$ da renda familiar. A associação entre o gasto catastrófico e as variáveis independentes foi testada por meio de regressão de Poisson. As prevalências de GCS variaram de 9,0\% a $11,3 \%$ e $18,9 \%$ a $24,4 \%$ nos limites de $10 \%$ e 25\%, sendo que o ano de 2011 apresentou os menores valores. A maior proporção dos gastos com saúde (94\%) foi relativa aos gastos com medicamentos. A prevalência de CGS foi menor entre responsáveis pelo domicílio com maior escolaridade quando comparados àqueles sem estudo nos limites de $10 \%$ e 25\%. Famílias com maior escore de riqueza apresentaram, nos dois limites, prevalência de GCS menores do que aquelas do primeiro quintil. Concluiu-se que os gastos com saúde afetaram significativamente o orçamento das famílias em Minas Gerais, sendo o gasto com medicamentos o principal componente dos gastos. Os achados reforçam o papel do SUS para minimizar o GCS e reduzir as desigualdades socioeconômicas.

Palavra-chave Gastos em saúde, Fatores socioeconômicos, Iniquidades em saúde 


\section{Introdução}

A garantia da proteção financeira das famílias quanto aos gastos em saúde é um dos grandes objetivos dos sistemas de saúde em todo o mundo'. Neste sentido as discussões sobre a eficiência dos sistemas de saúde e sua capacidade de proteção financeira têm sido cada vez mais recorrentes ${ }^{2,3}$.

Estimativas indicam que cerca de $100 \mathrm{mi}-$ lhões de pessoas no mundo são levadas à extrema pobreza a cada ano devido aos gastos em saúde ${ }^{4}$. No Brasil este cenário é particularmente preocupante. Apesar de o país possuir um sistema de saúde público, o Sistema Único de Saúde (SUS), e com pressupostos de universalidade, integralidade e equidade, a população ter a saúde como direito e garantida constitucionalmente, a participação das famílias nos gastos em saúde é expressiva. Prova disso é que o gasto privado ultrapassa o gasto público no Brasil. Em 2017 os gastos em saúde corresponderam a 9,2\% do Produto Interno Bruto, sendo que $58,3 \%$ foi relativo à participação das famílias, ou seja, percentual superior aos gastos públicos $(41,7 \%)^{5}$, padrão diferente do esperado para países que têm sistemas de saúde universais ${ }^{6}$.

Neste contexto, as famílias brasileiras, em grande parte, acabam arcando com seus gastos em saúde, impactando de forma importante na organização financeira familiar, inclusive fazendo com que elas incorram nos gastos catastróficos em saúde (GSC). Os GCS ocorrem quando os gastos em saúde correspondem a uma proporção importante (5\% a $40 \%)$ dos gastos totais de uma família, da sua renda ou de sua capacidade de pagamento, dependendo do método de cálculo ${ }^{7-15}$, ou seja, o GCS é uma medida de dificuldade financeira, causada pelos pagamentos em saúde ${ }^{2}$.

Estudos sobre os gastos catastróficos têm sido publicados em todo o mundo desde $1986^{16,17}$. Entretanto, ganhou destaque com o estudo de Xu et al..$^{12}$, publicado em 2003, que mostrou a situação dos GCS em 59 países.

Estimativas mais recentes, de 2010, apontam que 808 milhões de pessoas, $11,7 \%$ da população mundial, gastaram pelo menos $10 \%$ de seu orçamento familiar em serviços de saúde. Dessas pessoas, 179 milhões excederam $25 \%$ do seu orçamento familiar e 97 milhões empobreceram por causa de gastos com serviços de saúde ${ }^{14}$, sendo o impacto destes gastos significativamente maior sobre as famílias com menores condições socioeconômicas ${ }^{6,8,9}$. Em relação à prevalência de GCS entre os países, a porcentagem da população que excedeu os gastos com saúde em $10 \%$ ou $25 \%$ do seu orçamento familiar foi de $1,6 \%$ no Reino Unido, 2,6\% no Canadá e 24,8\% na Nigéria ${ }^{14}$.

No Brasil, Diniz et al..$^{18}$ com dados da Pesquisa de Orçamento Familiar (POF) de 1987/1988, 1995/1996 e 2002/2003 simularam diferentes pontos de corte para os GCS e identificaram uma prevalência que variou de $0,6 \%$ a $54,5 \%$. Barros et al. ${ }^{19}$, utilizando a POF de 2002/2003, simulando também diferentes pontos de corte observaram prevalências de $2 \%$ a $16 \%$. Já pesquisa mais recente, com dados da POF 2002/2003 e 2008/2009, identificou prevalência de GCS variando entre $0,7 \%$ e $25 \%$, com aumento das prevalências entre 2002/3 e 2008/9, tendo inclusive repercussão no aumento do percentual de famílias vivendo abaixo da linha de pobreza devido aos gastos em saúde ${ }^{7,9}$.

Assim, dentro deste contexto de estudos sobre GCS, fazer o seu monitoramento e avaliar a desigualdade socioeconômica são importantes para subsidiar gestores e pesquisadores para monitoramento, avaliação, bem como, na formulação de políticas e programas que objetivem diminuir sua prevalência. Além disso, são poucos os estudos sobre o tema no Brasil ${ }^{7,15,18-20}$ e estes se concentram em análises para o país ${ }^{718-20} \mathrm{e} / \mathrm{ou}$ considerando suas macrorregiões ${ }^{19,20}$, não existindo nenhum estudo que aborde a evolução dos GCS específico para o estado de Minas Gerais.

Assim, para contribuir nesta discussão, o objetivo deste estudo foi avaliar os gastos catastróficos e sua associação com condições socioeconômicas entre os anos de 2009 e 2013, utilizando a Pesquisa por Amostra de Domicílios de Minas Gerais (PAD-MG).

\section{Métodos}

Trata-se de um estudo transversal realizado com dados da Pesquisa por Amostra de Domicílios de Minas Gerais (PAD-MG) nos anos de 2009, 2011 e 2013. A PAD-MG é uma pesquisa amostral, domiciliar e com periodicidade bianual realizada no estado de Minas Gerais pela Fundação João Pinheiro, cujo objetivo principal é mostrar as peculiaridades regionais, além de fornecer uma ampla base de dados sobre vários aspectos socioeconômicos da população mineira, como: educação, saúde, trabalho, renda, consciência ambiental, gastos individuais e do domicílio, informações sobre a juventude e protagonismo juvenil para subsidiar a definição e avaliação anual de Políticas Públicas no Estado de Minas Gerais ${ }^{21}$. A coleta de dados ocorreu entre junho e novembro de 
2009, na PAD-MG 2009; entre outubro de 2011 e fevereiro de 2012, na PAD-MG 2011, e durante o ano de 2013, na PAD-MG 2013. O informante preferencial da pesquisa foi o(a) responsável pelo domicílio ou seu cônjuge ${ }^{22}$.

Nas três PAD-MG foram amostrados $18 \mathrm{mil}$ domicílios no estado de Minas Gerais ${ }^{21}$, sendo a amostra representativa para as doze mesorregiões, para as dez regiões de planejamento e para as regiões urbanas e rurais do estado. A amostragem em cada segmento urbano foi por conglomerado em dois estágios e em cada segmento rural em quatro estágios ${ }^{22}$. No primeiro estágio da amostragem do segmento urbano foram sorteados dois setores censitários, com reposição e probabilidade proporcional ao seu tamanho. No segundo estágio, foram selecionados por amostragem sistemática 15 domicílios em cada setor censitário sorteado. O sorteio do mesmo setor censitário duas vezes acarretaria na seleção de duas amostras de 15 domicílios sem reposição. No primeiro estágio da amostragem do segmento rural foram selecionados dois segmentos urbanos com reposição e probabilidade proporcional ao tamanho rural das cidades selecionadas nesse segmento. No segundo estágio, foi realizado um sorteio de uma das cidades selecionadas para a amostra, com reposição e probabilidade proporcional ao seu tamanho urbano. No terceiro estágio foi realizado um sorteio de um setor censitário rural de cada cidade sorteada, com reposição e probabilidade proporcional ao seu tamanho. No quarto estágio, selecionaram-se 15 domicílios por amostragem sistemática, em cada setor censitário sorteado. O sorteio do mesmo setor censitário duas vezes acarretaria na seleção de duas amostras de 15 domicílios sem reposição ${ }^{22}$. Um relato detalhado dos métodos pode ser encontrado na publicação da PAD-MG ${ }^{22}$. Neste estudo, foram utilizadas as PAD-MG de 2009, 2011 e 2013 resultando em 51.797 famílias pesquisadas, sendo incluídas 48.238 cujas informações para todas as variáveis de interesse estavam completas. Fizeram parte da amostra 15.924 famílias da PAD-MG de 2009, 17.053 famílias da PAG-MG de 2011 e 15.261 famílias da PAD-MG de 2013.

A variável dependente do presente estudo foi o Gasto Catastrófico em Saúde (sim e não), nos anos de 2009, 2011 e 2013. O GCS foi calculado de acordo com a metodologia proposta nos Objetivos de Desenvolvimento Sustentável e pela Organização Mundial de Saúde (OMS) ${ }^{23}$. O numerador foi composto pelo desembolso direto e o denominador pela renda familiar. Foram considerados catastróficos os gastos que ultrapassa- ram os limites de $10 \%$ e $25 \%$ da renda familiar ${ }^{23}$. O desembolso direto de cada domicílio foi composto pela soma dos pagamentos monetários efetuados em serviços referentes à saúde, como o gasto com atendimento médico, o gasto com internação, e o gasto com medicamentos, com período recordatório de 30 dias, exceto para o gasto com internação, que foi de 12 meses. Já a renda familiar foi calculada a partir da soma de todos os rendimentos da família (i.e. o rendimento mensal do trabalho e de outras fontes que não o trabalho $)^{22}$. Todas as variáveis relacionadas ao gasto e renda individual foram mensalizadas e corrigidas para o ano de $2013^{24}$. Para isso, utilizou-se o Índice de Preços ao Consumidor Amplo (IPCA) disponibilizado pelo IBGE, tendo como período base o referido ano, multiplicando-se os valores nominais de 2009 por 1,26 e os de 2011 por $1,12^{25}$.

As variáveis socioeconômicas do estudo foram anos de escolaridade do responsável pela família, categoriza em 0 a 3 anos; 4 a 7 anos; 8 a 11 anos; e mais de 11 anos de estudo e escore de riqueza categorizado em quintis, sendo o primeiro quintil o correspondente ao menor escore e o quinto quintil o correspondente ao maior escore. Este escore de riqueza foi construído com base em características e bens domiciliares ${ }^{26}$. Além dessas variáveis foram incorporadas na análise o sexo do responsável pela família (feminino ou masculino), a presença de idoso na família, a presença de dependentes com idade menor ou igual a 14 anos na família ${ }^{27,28}$, a posse de plano de saúde e o ano da PAD-MG (2009, 2011 e 2013).

Realizou-se a análise descritiva das variáveis independentes de acordo com os anos de estudo e estimou-se a prevalência de gastos catastróficos em saúde, para os pontos de corte de $10 \%$ e $25 \%$ da renda familiar. As diferenças entre proporções foram testadas por meio do teste quiquadrado com correção de Rao-Scott ${ }^{29}$. Estimou-se a proporção média de cada despesa com saúde em relação ao desembolso direto em cada ano de estudo. A associação entre o gasto catastrófico e as variáveis independentes foi testada por meio de regressão de Poisson univariada seguida de regressão múltivariável com estimação das razões prevalência (RP) e respectivos intervalos de confiança de 95\% (IC95\%). Os bancos de dados dos três anos de estudo foram agrupados e o ano de estudo utilizado como variável independente para testar as diferenças no gasto catastrófico entre os anos de estudo. A fim de se avaliar mudanças nas desigualdades socioeconômicas relacionadas ao gasto catastrófico, foi incluído um 
termo de interação entre os quintis de escore de riqueza e o ano de realização da PAD-MG e deste com a escolaridade do responsável pela família. A análise de dados foi realizada através do software Stata versão 14, considerando os pesos amostrais na análise.

\section{Resultados}

Em 2009, 61,3\% dos responsáveis pelos domicílios eram homens, 69,5\% possuíam entre 25 e 59 anos e 40,1\% dos responsáveis pelo domicílio possuíam 8 ou mais anos de estudo. A presença de dependentes na família foi relatada por $47 \%$ dos indivíduos, 28,6\% relataram presença de idosos no domicílio e $65,2 \%$ não tinham posse de plano de saúde. Em relação ao ano de 2009, houve diminuição na porcentagem da presença de dependentes na família $(42,5 \%)$, não houve alteração em relação a presença de idosos e a porcentagem de domicílios que não tinham posse de planos de saúde aumentou para 71,4\%. No ano de 2013, assim como nos anos anteriores, a porcentagem de mulheres responsáveis pelo domicílio foi menor quando comparada aos homens e a maioria tinha entre 25 e 59 anos. Em relação aos anos de estudo foi observada uma elevação na porcentagem $(42,3 \%)$ entre aqueles que tinham 8 anos ou mais de estudo quando comparada aos anos anteriores. Por fim, houve diminuição na porcentagem da presença de dependentes na família em relação a 2009, mas não houve alteração em relação a 2011, a porcentagem referente a presença de idosos foi a menor em relação aos anos anteriores e a posse de plano de saúde foi menor do que em 2011, mas maior em relação a 2009 (Tabela 1).

Em relação aos GCS, observou-se uma diminuição na prevalência entre 2009 e 2011 e um aumento entre 2011 e 2013 para os dois pontos de corte avaliados. A prevalência do GCS, segundo o ponto de corte de $\geq 10 \%$ da renda familiar, diminuiu de 24,4\% em 2009 para 18,9\% em 2011 e aumentou para 21,4\% em 2013. Quando empregado o ponto de corte de $\geq 25 \%$ da renda familiar, observou-se uma diminuição de 11,3\% em 2009 para 9,0\% em 2011 e um aumento para 11,2\% em 2013 (Tabela 2). No que se refere às despesas com saúde, os gastos com medicamentos representaram aproximadamente $94 \%$ do desembolso direto em todos os anos (Tabela 3 ).

$\mathrm{Na}$ análise univariada, tanto no ponto de corte de $\geq 10 \%$ da renda familiar quanto no de $\geq 25 \%$ da renda familiar, os domicílios que tinham como responsáveis os homens, os mais escolari- zados e possuíam o maior quintil de renda apresentaram uma menor probabilidade de ter GCS. Por outro lado, a maior probabilidade de GCS foi observada entre os responsáveis que tinham 60 anos ou mais [RP 1,75 (IC95\% 1,54-1,98) e RP 1,33 (IC95\% 1,12-1,59), respectivamente, para os pontos de corte de $10 \%$ e $25 \%$ ], onde havia presença de dependentes [RP 1,11 (IC95\% $1,03-1,19)$ para o ponto de corte de $25 \%$ ] e de idosos na família [RP 1,62 (IC95\% 1,56-1,70) e RP 1,33 (IC95\% 1,24-1,42), respectivamente, para os pontos de corte de $10 \%$ e $25 \%$ ] e entre aqueles que tinham posse de planos de saúde [RP 1,10 (IC95\% 1,05-1,16) para o ponto de corte de $10 \%]$. Além disso, observou-se que a prevalência de GCS foi maior nos anos de 2009 e 2013 quando comparada ao ano de 2011 nos dois pontos de cortes avaliados (Tabela 4 ).

A análise ajustada sugeriu associações semelhantes entre a prevalência de GCS no ponto de corte de $10 \%$ e 25\%. Famílias cujos responsáveis possuíam 12 ou mais anos de estudo apresentaram menores prevalências de GCS do que aquelas cujo responsável não estudou [RP 0,65 (IC95\% 0,57-0,73) e RP 0,68 (IC95\% 0,56-0,84), respectivamente, para os pontos de corte de $10 \%$ e 25\%]. Associação inversa entre a prevalência de GCS também foi observada em relação aos quintis de riqueza. Famílias pertencentes ao quinto quintil, apresentaram, nos limites de $10 \%$ e $25 \%$ respectivamente, prevalência de gastos catastróficos $14 \%$ e $27 \%$ menores do que as famílias do primeiro quintil. Verificou-se que para os dois pontos de corte estudados, houve uma redução da prevalência entre 2009 e 2011 e posterior aumento entre 2011 e 2013 (Tabela 5). Não houve interação entre os quintis de escore de riqueza ou da escolaridade do responsável pela família com os anos da pesquisa, sugerindo que as diferenças socioeconômicas se mantiveram inalteradas no período.

\section{Discussão}

Este estudo analisou a evolução do GCS entre 2009 e 2013 e a associação desse gasto com as condições socioeconômicasno estado de Minas Gerais, Brasil. Os resultados mostraram que houve diminuição na prevalência do GCS entre 2009 e 2011 e aumento entre os anos de 2011 e 2013. Além disso, foi observada que as famílias pertencentes ao quintil mais rico tiveram prevalências menores de GCS,quando comparadas ao quintil de menor renda. 
Estudo conduzido na Coreia do Sul ${ }^{30}$ utilizando no numerador o desembolso direto com saúde e no denominador a capacidade de pagamento das famílias, que é o gasto da família menos os gastos realizados com alimentação, reportou uma prevalência igual a $24,3 \%$ em 2012 e a

Tabela 1. Distribuição da amostra. PAD-MG, 20092013.

\begin{tabular}{|c|c|c|c|}
\hline & $\begin{array}{c}2009 \\
(\%)\end{array}$ & $\begin{array}{c}2011 \\
(\%)\end{array}$ & $\begin{array}{c}2013 \\
(\%)\end{array}$ \\
\hline \multicolumn{4}{|l|}{ Sexo do responsável } \\
\hline Feminino & 38,7 & 39,0 & 33,9 \\
\hline Masculino & 61,3 & 61,0 & 66,1 \\
\hline \multicolumn{4}{|l|}{ Idade do responsável } \\
\hline 18 a 24 anos & 5,6 & 4,5 & 5,4 \\
\hline 25 a 59 anos & 69,5 & 70,6 & 70,5 \\
\hline 60 anos e + & 24,9 & 24,9 & 24,1 \\
\hline \multicolumn{4}{|c|}{ Anos de estudo do responsável } \\
\hline 0 & 9,8 & 10,2 & 10,8 \\
\hline 1 a 3 anos & 15,7 & 18,4 & 16,8 \\
\hline 4 a 7 anos & 34,4 & 32,0 & 30,1 \\
\hline 8 a 11 anos & 31,1 & 30,7 & 33,2 \\
\hline Mais de 11 anos & 9,0 & 8,7 & 9,1 \\
\hline \multicolumn{4}{|c|}{$\begin{array}{l}\text { Presença de dependentes na } \\
\text { família }\end{array}$} \\
\hline Sim & 47,0 & 42,5 & 42,9 \\
\hline Não & 53,0 & 57,5 & 57,1 \\
\hline \multicolumn{4}{|c|}{ Presença de idoso na família } \\
\hline Sim & 28,6 & 28,9 & 27,1 \\
\hline Não & 71,4 & 71,1 & 72,9 \\
\hline \multicolumn{4}{|c|}{ Posse de plano de saúde } \\
\hline Sim & 34,8 & 28,6 & 31,0 \\
\hline Não & 65,2 & 71,4 & 69,0 \\
\hline
\end{tabular}

20,4\% em 2013 utilizando o ponto de corte de $10 \%$. Em Uganda, Kwesiga et al. ${ }^{31}$ consideraram no cálculo do GCS o desembolso direto com gastos relacionados à saúde no numerador e o consumo das famílias no denominador, encontrando, no limite de $10 \%$, uma prevalência de $22,8 \%$ em 2009/2010. No Brasil, Boing et al. ${ }^{7}$ definiram GCS por meio de diferentes métodos de cálculo: $10,0 \%$ e $20,0 \%$ dos gastos totais em saúde dividido pelo total de despesas do domicílio e 40,0\% do desembolso direto dividido pela capacidade de pagamento da família. Os autores mostraram que as prevalências do GCS variaram de $0,7 \%$ a $21,1 \%$ em $2002-2003$ e de $1,4 \%$ a $25 \%$ em 2008 2009 a depender da definição e ponto de corte do desfecho ${ }^{7}$. Entretanto, os métodos utilizados para avaliar o GCS nos diferentes estudos são distintos, dificultando a comparação direta dos achados.

Apesar da prevalência do GCS ter diminuído entre 2009 e 2011, houve um aumento entre 2011 e 2013. Entre as possíveis explicações para esta variação poderiam ser consideradas as alterações sofridas na economia brasileira nesse período, o financiamento público de medicamento e a cobertura da atenção primária em saúde. Entre 2000 e 2010 houve um crescimento econômico que foi marcado pela redução significativa das desigualdades de renda e sociais ${ }^{32}$. No entanto, a partir de 2011, a economia brasileira começou a desacelerar, sofreu uma recessão prolongada que foi acompanhada pela piora de indicadores sociais $^{5}$ que podem ter contribuído para o aumento da prevalência do GCS. Em relação ao financiamento de medicamentos, houve uma a redução dos repasses dos entes federativos para após o ano de $2010^{33}$. Já a atenção primária em saúde

Tabela 2. Proporção de gastos catastróficos nos três anos da pesquisa. PAD-MG, 2009-2013.

\begin{tabular}{rccc}
\hline \multirow{2}{*}{ Gasto catastrófico } & PAD-MG 2009 & PAD MG 2011 & PAD-MG 2013 \\
\cline { 2 - 4 } & \%(IC95\%) & \% (IC95\%) & \% (IC95\%) \\
\hline$\geq 10 \%$ da renda familiar & $24,4(23,3-25,3)$ & $18,9(18,2-19,6)$ & $21,4(20,5-22,3)$ \\
$\geq 25 \%$ da renda familiar & $11,3(10,6-12,0)$ & $9,0(8,5-9,5)$ & $11,2(10,5-11,9)$ \\
\hline
\end{tabular}

Fonte: Autores.

Tabela 3. Proporção média das despesas de saúde em relação ao desembolso direto. PAD-MG, 2009-2013.

\begin{tabular}{lccc}
\hline \multicolumn{1}{c}{ Desembolso direto } & PAD-MG 2009 (\%) & PAD MG 2011 (\%) & PAD-MG 2013 (\%) \\
\hline Consulta & $5,3(4,9-5,7)$ & $5,0(4,5-5,5)$ & $4,9(4,5-5,4)$ \\
Internação & $0,9(0,8-1,1)$ & $1,4(1,2-1,6)$ & $1,6(1,2-1,9)$ \\
Medicamento & $93,8(93,3-94,2)$ & $93,6(93,1-94,1)$ & $93,5(92,9-94,1)$ \\
\hline
\end{tabular}


Tabela 4. Análises univariadas entre o gasto catastrófico e as variáveis independentes. PAD-MG, 2009-2013.

\begin{tabular}{|c|c|c|}
\hline \multirow{3}{*}{ Variáveis } & \multicolumn{2}{|c|}{ Desembolso direto } \\
\hline & $\geq 10 \%$ & $\geq 25 \%$ \\
\hline & RP (IC95\%) & RP (IC95\%) \\
\hline \multicolumn{3}{|l|}{ Sexo do responsável } \\
\hline Feminino & 1 & 1 \\
\hline Masculino & $0,85(0,82-0,89)^{\star * *}$ & $0,84(0,78-0,90)^{\star * *}$ \\
\hline \multicolumn{3}{|l|}{ Idade do responsável } \\
\hline 18 a 24 anos & 1 & 1 \\
\hline 25 a 59 anos & $1,09(0,96-1,24)$ & $0,99(0,83-1,18)$ \\
\hline $60+$ anos & $1,75(1,54-1,98)^{\star * *}$ & $1,33(1,12-1,59)^{\star *}$ \\
\hline \multicolumn{3}{|l|}{ Anos de estudo do responsável } \\
\hline Não estudou & 1 & 1 \\
\hline 1 a 3 anos & $0,91(0,85-0,98)^{\star}$ & $0,95(0,84-1,08)$ \\
\hline 4 a 7 anos & $0,77(0,72-0,83)^{\star \star *}$ & $0,82(0,73-0,92)^{* *}$ \\
\hline 8 a 11 anos & $0,65(0,61-0,70)^{\star * \star}$ & $0,69(0,61-0,78)^{\star * *}$ \\
\hline $12+$ anos & $0,54(0,48-0,60)^{\star \star \star \star}$ & $0,56(0,46-0,67)^{\star * \star}$ \\
\hline \multicolumn{3}{|l|}{ Quintil de bens riqueza } \\
\hline $1^{\circ}$ Quintil (menor) & 1 & 1 \\
\hline $2^{\circ}$ Quintil & $1,01(0,95-1,08)$ & $0,98(0,88-1,08)$ \\
\hline $3^{\circ}$ Quintil & $0,98(0,91-1,05)$ & $0,95(0,86-1,06)$ \\
\hline $4^{\circ}$ Quintil & $0,91(0,85-0,98)^{\star}$ & $0,80(0,72-0,90)^{* * *}$ \\
\hline $5^{\circ}$ Quintil & $0,82(0,76-0,88)^{* * *}$ & $0,68(0,60-0,76)^{* * *}$ \\
\hline \multicolumn{3}{|c|}{ Presença de dependentes na família } \\
\hline Não & 1 & 1 \\
\hline Sim & $1,00(0,96-1,05)$ & $1,11(1,03-1,19)^{\star *}$ \\
\hline \multicolumn{3}{|l|}{ Presença de idoso na família } \\
\hline Não & 1 & 1 \\
\hline Sim & $1,62(1,56-1,70)^{* * *}$ & $1,33(1,24-1,42)^{* * *}$ \\
\hline \multicolumn{3}{|l|}{ Posse de plano de saúde } \\
\hline Não & 1 & 1 \\
\hline Sim & $1,10(1,05-1,16)^{* * *}$ & $0,97(0,91-1,08)$ \\
\hline \multicolumn{3}{|l|}{ Ano } \\
\hline 2009 & $1,29(1,23-1,36)^{\star \star \star *}$ & $1,25(1,16-1,36)^{* * *}$ \\
\hline 2011 & 1 & 1 \\
\hline 2013 & $1,13(1,07-1,20)^{\star \star \star}$ & $1,24(1,14-1,35)^{\star * *}$ \\
\hline
\end{tabular}

Fonte: Autores.

contou com uma expansão de sua cobertura, no Brasil e em MG a partir de $2006^{34}$. O aumento da cobertura na atenção primária pode aumentar o acesso a consulta médica, que é uma parte do gasto em saúde, mas a redução do repasse destinado ao gasto com o medicamento teria um impacto significativamente maior.

No presente estudo, o medicamento foi responsável por $94 \%$ do desembolso direto com saúde das famílias nos três anos analisados.Esses achados podem refletir a dificuldade do acesso aos medicamentos devido à baixa disponibilida- de no sistema público de saúde brasileiro (SUS) ${ }^{7}$. Análise realizada com dados da Pesquisa Nacional por Amostra de Domicílios (PNAD) de 2008 verificou que $54,7 \%$ dos usuários do SUS, que receberam prescrição medicamentosa no sistema, não obtiveram todos os medicamentos gratuitamente ${ }^{35}$. Posteriormente, um levantamento realizado pelo IBGE por meio da Pesquisa Nacional de Saúde (PNS) em 2013, mostrou que a proporção de pessoas que não conseguiram obter nenhum medicamento receitado no serviço público de saúde foi de $66,8 \%{ }^{36}$. Assim, grande parte dos 
Tabela 5. Razões de prevalências ajustadas para os fatores associados aos gastos catastróficos em saúde. PADMG, 2009-2013.

\begin{tabular}{|c|c|c|}
\hline & \multicolumn{2}{|c|}{ Gasto catastrófico em saúde } \\
\hline & $\geq 10 \%$ & $\geq \mathbf{2 5} \%$ \\
\hline & RP (IC95\%) & RP (IC95\%) \\
\hline \multicolumn{3}{|c|}{ Sexo responsável (feminino) } \\
\hline Masculino & $0,90(0,86-0.94)^{* * *}$ & $0,86(0,80-0,92)^{\star * *}$ \\
\hline \multicolumn{3}{|c|}{ Escolaridade (não estudou) } \\
\hline $1-3$ anos & $1,00(0,92-1,07)$ & $1,02(0,90-1,17)$ \\
\hline 4-7 anos & $0,91(0,85-0,99)^{\star}$ & $0,93(0,81-1,05)$ \\
\hline $8-11$ anos & $0,81(0,75-0,89)^{* * *}$ & $0,82(0,71-0,94)^{\star *}$ \\
\hline $12+$ anos & $0,65(0,57-0,73)^{* * *}$ & $0,68(0,56-0,84)^{\star * *}$ \\
\hline \multicolumn{3}{|c|}{ Quintil de bens de riqueza ( $1^{\circ}$ quintil) } \\
\hline $2^{\circ}$ quintil & $1,02(0,96-1.09)$ & $0,99(0,89-1,09)$ \\
\hline $3^{\circ}$ quintil & $0,99(0.93-1,06)$ & $0,97(0,88-1,08)$ \\
\hline $4^{\circ}$ quintil & $0,94(0,87-1,01)$ & $0,83(0,74-0,93)^{\star *}$ \\
\hline $5^{\circ}$ quintil (maior) & $0,86(0,79-0,93)^{* * *}$ & $0,73(0,64-0,83)^{* * *}$ \\
\hline \multicolumn{3}{|l|}{ Ano de estudo (2011) } \\
\hline 2009 & $1,29(1,22-1,35)^{* * *}$ & $1,25(1,15-1,36)^{\star * *}$ \\
\hline 2013 & $1,15(1,09-1,21)^{\star * *}$ & $1,26(1,16-1,36)^{\star * *}$ \\
\hline \multicolumn{3}{|l|}{ Idoso na família } \\
\hline Sim & $1,48(1,41-1,56)^{\star * *}$ & $1,21(1,11-1,31)^{\star * *}$ \\
\hline \multicolumn{3}{|l|}{ Posse de plano de saúde } \\
\hline Sim & $1,22(1,15-1,29)^{* * *}$ & $1,14(1,05-1,24)^{\star *}$ \\
\hline
\end{tabular}

Fonte: Autores.

usuários que não recebem os medicamentos pelo SUS acabam adquirindo-os por meio de compra no setor privado ${ }^{35}$. Além disso, cabe destacar que como envelhecimento da população e o aumento da prevalência de doenças crônicas no Brasil ${ }^{37}$ espera-se uma tendência crescente do GCS caso o acesso aos medicamentos não seja ampliado no SUS, uma vez que aproximadamente $75 \%$ da população é dependente do sistema público de saúde ${ }^{38}$.

Os resultados sugerem importantes desigualdades socioeconômicas em relação ao GCS, conforme observado previamente no Brasil $^{7}$ e em outros países ${ }^{39-43}$. A prevalência do GCS foi maior entre as famílias nas quais o chefe era menos escolarizado e entre os menores quintis de riqueza. Piores condições socioeconômicas comprometem o acesso à saúde de diferentes formas, desde o nível de percepção dos problemas de saúde e capacidade de entendimento das informações repassadas até o consumo e utilização dos serviços de saúde ${ }^{44,45}$. Indivíduos nas menores posições socioeconômicas também apresentam piores condições de saúde ${ }^{7,46}$ e, consequentemente, maior necessidade do uso e consumo de bens e serviços de saúde. Assim, os problemas enfrentados pelo sistema de saúde brasileiro para garantir acesso aos serviços e suprir as demandas de saúde da população força os indivíduos a incorrerem em gastos com saúde, acessando serviços privados ${ }^{7}$. Estes gastos afetam principalmente os mais pobres, pois apesar dos mais ricos terem um gasto absoluto em saúde maior, a proporção desses gastos em relação à renda é mais elevada entre aqueles de menor renda, resultando no GCS ${ }^{7,9,41-43}$.

Finalmente cabe destacar que os achados sugerem que a posse de plano de saúde foi associada a maior probabilidade de GCS. Resultados heterogêneos são observados em relação a este fator, sendo considerado protetor do GCS em alguns países $^{47,48}$ e fator de risco em outros ${ }^{49,50}$, incluindo o Brasil ${ }^{7,19}$. A posse do plano de saúde pode ser considerada um proxy das condições socioeconômicas e é um gasto previsto no orçamento familiar entre os mais ricos ${ }^{7}$. Porém, sabe-se que o sistema de copagamento entre os mais pobres é cada vez mais habitual no Brasil em função das limitações do SUS, e muitas vezes representa um 
valor superior à capacidade de pagamento desses indivíduos ${ }^{7}$. Dados da Agência Nacional de Saúde Suplementar, de junho de 2020, indicam que a maior parte da população possui planos de saúde do tipo empresarial ${ }^{51}$, ou seja, por serem funcionários de uma empresa têm direito ao benefício, sem custo ou com um custo parcial. Entretanto, muitas vezes esta população apesar de ter acesso aos serviços de saúde não tem condições de arcar com outras despesas provenientes dos serviços de saúde que utilizou. A posse de plano de saúde, além de causar por si só impacto no orçamento familiar desses indivíduos, quando precisam despender valores para o seu pagamento, não impede outros gastos com saúde, já que muitos planos têm cobertura limitada e não cobrem despesas com alguns serviços especializados e com medicamentos ${ }^{7,19}$.

Este estudo apresenta vantagens e limitações. A principal vantagem é que este foi o primeiro estudo a relatar o GCS e a magnitude de sua associação com as condições socioeconômicas em Minas Gerais. A utilização das bases de dados de estudos que empregaram os mesmos métodos e a representativa da amostra para população de Minas Gerais também são pontos fortes deste estudo e permitiram a comparação entre os períodos gerando um panorama para o Estado. Além disso, foi realizado com dados da PAD-MG que é uma grande pesquisa domiciliar realizada no Estado. As limitações incluem a falta de consenso sobre as formas de cálculo e pontos de corte para mensurar o GCS, o que dificulta a comparação dos resultados. Outra limitação é o caráter transversal do estudo, que não permite o estabelecimento de uma relação causal entre a GCS e os fatores investigados. O período recordatório das medidas de gastos em saúde e o fato de das informações serem autorrelatadas podem ter gerado algum viés na estimativa da despesa. Entretanto, este método tem sido empregado em diversos estudos nacionais, como a $\mathrm{POF}^{24}$ e a Pesquisa Nacional por Amostra de Domicílios Contínua (PNAD) ${ }^{52}$.

Apesar de ter sido observada uma melhora entre os anos de 2009 e 2011, a prevalência de GCS voltou a subir entre 2011/2013, sendoos gastos com medicamentos o maior impacto no desembolso direto em todos os anos. Além disso, desigualdades socioeconômicas foram evidenciadas no GCS. Esses achados mostram a necessidade de avaliação das políticas de acesso aos serviços e bens de saúde, especialmente aos medicamentos. Além disso, reforçam o papel do SUS para minimizar o GCS e reduzir as desigualdadessocioeconômicas. Novos estudos devem monitorar continuamente os efeitos da crise econômica, que teve início em 2014, sobre os gastos com saúde no Brasil e no estado de Minas Gerais, a fim de fornecer subsídios relevantes para as políticas de saúde.

\section{Colaboradores}

JB Macedo participou da elaboração e revisão do manuscrito, interpretação dos resultados, aprovação da versão final. AC Boing participou da concepção e planejamento do estudo, interpretação dos resultados, elaboração e revisão do manuscrito, aprovação da versão final. JM Andrade participou da interpretação dos resultados, elaboração e revisão do manuscrito, aprovação da versão final. H Saulo participou da análise e interpretação dos resultados, revisão do manuscrito, aprovação da versão final. RN Fernandez participou da análise e interpretação dos resultados, revisão do manuscrito, aprovação da versão final. FB Andrade participou da concepção e planejamento do estudo, análise e interpretação dos resultados, elaboração e revisão do manuscrito, aprovação da versão final.

\section{Agradecimentos}

Este estudo foi financiado pela Fundação de Amparo à Pesquisa do Estado de Minas Gerais (FAPEMIG). 


\section{Referências}

1. Organização Mundial da Saúde (OMS). Relatório Mundial da Saúde 2010: Financiamento dos Sistemas de Saúde - o caminho para a Cobertura Universal. Genebra: OMS; 2010.

2. Wagstaff A, Flores G, Hsu J, Smitz MF, Chepynoga, Buisman LR, Wilgenburg KV, Eozenou P. Progress on catastrophic health spending in 133 countries: a retrospective observational study. Lancet Glob Health 2018; 6(2):e169-e179.

3. Wagstaff A, Neelsen S. A comprehensive assessment of universal health coverage in 111 countries: a retrospective observational study. Lancet Glob Health 2020; 8(1):e39-e49.

4. World Health Organization (WHO). Public Spendingon Health: A Closer Look at Global Trends. Geneva: WHO; 2018.

5. Instituto Brasileiro de Geografia e Estatística (IBGE). Sistemas de contas nacionais trimestrais - SCNT [Internet]. [acessado 2019 out 21]. Disponível em: https:// www.ibge.gov.br/estatisticas/economicas/contas-nacionais/9300-contas-nacionais-trimestrais.html? $=\&-$ $\mathrm{t}=$ series-historicas\&utm_source $=$ landing\&utm_medium=explica\&utm_campaign=pib\#evolucao-taxa.

6. Figueiredo JO, Prado NMBL, Medina MGP, Silva J. Gastos público e privado com saúde no Brasil e países selecionados. Saude Debate 2018; 42(2):37-47.

7. Boing AC, Bertoldi AD, Barros AJD, Posenato LG, Peres KG. Socioeconomic inequality in catastrophic health expenditure in Brazil. Rev Saude Publica 2014; 48(4):632-641.

8. Buigut S, Ettarh R, Amendah DD. Catastrophic health expenditure and its determinants in Kenya slum communities. Int J Equity Health 2015; 14:46.

9. Boing AC, Bertoldi AD, Posenato LG, Peres KG. The influence of health expenditures on household impoverishment in Brazil. Rev Saude Publica 2014; 48(5):797-807.

10. Adhikari SR, Maskay NM, Sharma BP. Paying for hospital-based care of Kala-azar in Nepal: assessing catastrophic, impoverishment and economic consequences. Health Policy Plan 2009; 24(2):129-139.

11. Damme WV, Leemput LV, Por I, Hardeman W, Meessen B. Out-of-pocket health expenditure and debt in poor households: evidence from Cambodia. Trop MedInt Health 2004; 9(2):273-280.

12. Xu K, Evans DB, Kawabata K, Zeramdini R, Murray CJL.Household catastrophic health expenditure: a multicountry analysis. Lancet 2003; 362(9378):111117.

13. Wagstaff A, Doorslaer EV. Catastrophe and impoverishment in paying for health care: with applications to Vietnam 1993-1998. Health Econ 2003; 12(11):921933.

14. World Health Organization (WHO). World health statistics 2018: monitoring health for the SDGs, sustainable development goals. Geneva: WHO; 2018.

15. Barros AJ, Bertoldi AD. Out-of-pocket health expenditure in a population covered by the Family Health Program in Brazil. Int J Epidemiol 2008; 7(4):758-765.

16. Berki SE. A look at catastrophic medical expenses and the poor. Health Affairs 1986; 5(4):138-45.

17. Wyszewianski L. Financially catastrophic and highcost cases: definitions, distinctions, and their implication for policy formulation. Inquiry 1986; 23:382-394.
18. Diniz BPC, Servo LMS, Piola SF, Eirado M. Gasto das famílias com saúde no Brasil: evolução e debate sobre gasto catastrófico. In: Silveira FG, Servo LM, Menezes, Piola SF, organizadores. Gasto e consumo das famílias brasileiras contemporâneas. Brasília: Ipea; 2007. p. 143166.

19. Barros AJD, Bastos JL, Dâmaso AH. Catastrophic spending on health care in Brazil: private health insurance does not seem to be the solution. Cad Saude Publica 2011; 27:s254-s262.

20. Diaz MDM, Sarti FM, Campino ACC, Iunes R. Catastrophic Health Expenditure in Brazil: Regional Differences, Budget Constraints and Private Health Insurance. In: Knaul FM, Wong R, Arreola-Ornelas H. Household Spending and Impoverishment. Volume 1 of Financing Health in Latin America Series. Cambridge: Harvard Global Equity Initiative, in collaboration with Mexican Health Foundation and International Development Research Centre; 2012.

21. Pesquisa por Amostra de Domicílios de Minas Gerais (PAD-MG). Boletim PAD-MG. Belo Horizonte: Fundação João Pinheiro, Centro de Estatística e Informações; 2014.

22. Pesquisa por Amostra de Domicílios de Minas Gerais (PAD-MG). Plano amostral, métodos de ponderação e metodologia. Belo Horizonte: Fundação João Pinheiro, Centro de Estatística e Informação; 2010.

23. World Health Organization (WHO). The World Bank. Tracking Universal Health Coverage: 2017 Global Monitoring Report. Geneva: WHO; 2017.

24. Instituto Brasileiro de Geografia e Estatística (IBGE). Pesquisa de Orçamentos Familiares 2008-2009: despesas, rendimentos e condições de vida. Rio de Janeiro: IBGE; 2010.

25. Feijó CA, Ramos RLO. Contabilidade social: referência atualizada das contas nacionais do Brasil. $5^{\mathrm{a}}$ ed. Rio de Janeiro: Elsevier; 2017.

26. Fry K, Firestone R, Chakraborty NM. Measuring Equity with Nationaly Representative Wealth Quintiles. Washington, D.C.: PSI; 2014.

27. United Nations (UN). United Nations DemographicYearbook review: National reporting of age and sex-specific data Implications for international recommendations. New York: UN; 2004.

28. Nery PF, Tenoury GN, Shikida C. Probabilidade de desemprego por faixa etária: implicações para idade mínima e políticas de emprego. Brasília: Núcleo de Estudos e Pesquisas/CONLEG/Senado; 2018.

29. Rao JNK, Scott AJ. The analysis of categorical data from complex sample surveys: chi-squared tests for goodness of fit the independence in two-way tables. $J$ Am Stat Assoc 1981; 76:221-230.

30. Kang JH, Kim CW. Relationship Between Catastrophic Health Expenditures and Income Quintile Decline. Osong Public Health Res Perspec 2018; 9(2):73-80.

31. Kwesiga B, Zikusooka CM, Ataguba JE. Assessing catastrophic and impoverishing effects of health care payments in Uganda. BMC Health Serv Res 2015; 15:30.

32. Instituto de Pesquisa Econômica Aplicada (Ipea). IPEA data. Emprego e renda [Internet]. [acessado 2019 out 21]. Disponível em: http://www.ipeadata.gov.br/ Default.aspx. 
33. Vieira FS. Evolução do gasto com medicamentos do Sistema Único de Saúde no período de 2010 a 2016. Texto para discussão. Rio de Janeiro: Ipea; 2018.

34. Neves RG, Flores TR, Duro SMS, Nunes BP, Tomasi E. Tendência temporal da cobertura da Estratégia Saúde da Família no Brasil, regiões e Unidades da Federação, 2006-2016. Epidemiol Serv Saude 2018 27(3):e2017170.

35. Boing AC, Bertoldi AD, Boing AF, Bastos JL, Peres KG Acesso a medicamentos no setor público: análise de usuários do Sistema Único de Saúde no Brasil. Cad Saude Publica 2013; 29(4):691-701.

36. Instituto Brasileiro de Geografia e Estatística (IBGE) Pesquisa Nacional de Saúde (PNS): 2013: acesso e utilização dos serviços de saúde, acidentes e violências: Brasil, grandes regiões e unidades da federação. Rio de Janeiro: IBGE; 2015.

37. Lima-Costa MF, Andrade FB, Souza Junior PRB, Neri AL, Duarte YAO, Castro-Costa E, Oliveira C. The Brazilian Longitudinal Study of Aging (ELSI-Brazil): Objectives and Design. Am J Epidemiol 2018; 187(7):1345-1353.

38. Mendes EV. 25 anos do Sistema Único de Saúde: resultados e desafios. Estud Av 2013; 27(78):27-34.

39. Su TT, Kouyaté B, Steffen S. Catastrophic household expenditure for health care in a lowincome society: a study from Nouna District, Burkina Faso. Bull World Health Organ 2006; 84:21-27.

40. Li Y, Wu Q, Xu L, Legge D, Hao Y, Gao L, Ning N, Wan G. Factors affecting catastrophic health expenditure and impoverishment from medical expenses in China: policy implications of universal health insurance. Bull World Health Organ 2012; 90(9):664-671.

41. Séne LM, Cissé M. Catastrophic out-of-pocket payments for health and poverty nexus: evidence from Senegal. Int J Health Econ Manag 2015; 15(3):307-328

42. Tomini SM, Packard TG, Tomini F. Catastrophic and impoverishing effects of out-of-pocket payments for health care in Albania: evidence from Albania Living Standards Measurement Surveys 2002, 2005 and 2008. Health Policy and Plan 2013; 28(4):419-428.

43. Sanmartin C, Hennessy D, Lu Y, Law MR. Trends in out-of-pocket health care expenditures in Canada, by household income, 1997 to 2009. Health Reps 2014; 25(4):13-17.

44. Silva ZP, Ribeiro MCSA, Barata RB, Almeida MF Perfil sociodemográfico e padrão de utilização dos serviços de saúde do Sistema Único de Saúde (SUS), 2003-2008. Cien Saude Colet 2011; 16(9):3807-3816.
45. Barata RB. Como e por que as desigualdades sociais $\mathrm{fa}$ zem mal à saúde. Rio deJaneiro: Editora Fiocruz; 2009.

46. Aregbeshola BS, Khan SM. Determinants of catastrophic health expenditure in Nigeria. Eur J Health Econ 2018; 19(4):521-532.

47. Kavosi Z, Rashidian A, Pourreza A, Majdzadeh R, Pourmalek F, Hosseinpour AR, Mohammad K, Arab M. Inequality in household catastrophic health care expenditure in a low-income society of Iran. Health Policy Plan 2012; 27(7):613-623.

48. Knaul FM, Wong R, Arreola-Ornelas H, Méndez O, Bitrán R, Campino ACC, Flórez Nieto CE, Iunes Fontes R, Gledion U, Maceira D, Rathe M, Valdivia M, Vargas JR, Pleic M, Diaz JJ, Diaz MDM, Valdés W, Carmona RV, Zúñiga MP, Lafontaine L, Muñoz R, Pardo R, Reynoso AM, Santana MI, Vidarte R. Household catastrophic health expenditures: A comparative analysis of twelve Latin American and Caribbean Countries. Salud Publica Mex 2011; 53(Supl. 2):s85-s95.

49. Ekman B. Catastrophic health payments and health insurance: Some counterintuitive evidence from one low-income country. Health Policy 2007; 83(2-3):304313.

50. Himmelstein DU, Thorne D, Warren W, Woolhandler S. Medical bankruptcy in the United States, 2007: results of a nationalstudy. Am J Med 2009; 122(8):741746.

51. Agência Nacional de Saúde Suplementar. Dados Gerais [Internet]. [acessado 2020 out 21]. Disponível em: http://www.ans.gov.br/perfil-do-setor/dados-gerais.

52. Instituto Brasileiro de Geografia e Estatística (IBGE) Um panorama da saúde no Brasil: acesso e utilização dos serviços, condiç̃es de saúde e fatores de risco e proteção à saúde, 2008. Rio de Janeiro: IBGE; 2001.

Artigo apresentado em 28/08/2020

Aprovado em 21/11/2020

Versão final apresentada em 23/11/2020

Editores-chefes: Romeu Gomes, Antônio Augusto Moura da Silva 